\title{
NEW APPROACH FOR WOLFE'S MODIFIED SIMPLEX METHOD TO SOLVE QUADRATIC PROGRAMMING PROBLEMS
}

\author{
Kirtiwant P. Ghadle ${ }^{1}$, Tanaji S. Pawar ${ }^{2}$ \\ ${ }^{l}$ Department of Mathematics, Dr. Babasaheb Ambedkar Marathwada University, Aurangabad, Maharashtra, India \\ ${ }^{2}$ Department of Mathematics, Dr. Babasaheb Ambedkar Marathwada University, Aurangabad, Maharashtra, India
}

\begin{abstract}
In this paper, an alternative method for Wolfe's modified simplex method is introduced. This method is easy to solve quadratic programming problem $(Q P P)$ concern with non-linear programming problem (NLPP). In linear programming models, the characteristic assumption is the linearity of the objective function and constraints. Although this assumption holds in numerous practical situations, yet we come across many situations where the objective function and some or all of the constraints are nonlinear functions. The non-linearity of the functions makes the solution of the problem much more involved as compared to LPPs and there is no single algorithm like the simplex method, which can be employed to solve efficiently all NPPs.
\end{abstract}

Keywords: Quadratic programming problem, New approach, Modified simplex method, and Optimal solution.

\section{INTRODUCTION}

Quadratic programming problems (QPP) deals with the nonlinear programming problem (NLPP) of maximizing (or minimizing) the quadratic objective function subject to a set of linear inequality constraints.

In general QPP be:

$$
\operatorname{Maximize} Z=f(x)=\sum_{j=1}^{n} c_{j} x_{j}+\frac{1}{2} \sum_{j=1}^{n} \sum_{k=1}^{n} c_{j k} x_{j} x_{k}
$$

Subject to the constraints:

$$
\sum_{j=1}^{n} a_{i j} x_{j} \leq b_{i}, x_{j} \geq 0(\mathrm{i}=1,2, \ldots, \mathrm{m}, \mathrm{j}=1,2, \ldots, \mathrm{n})
$$

where $c_{j k}=c_{k j}$ for all $j, k$ and $b_{i} \geq 0$ for all $i=1,2, \ldots, m$.

Also, assume that the quadratic form

$$
\sum_{j=1}^{n} \sum_{k=1}^{n} c_{j k} x_{j} x_{k}
$$

be negative semi-definite.

Terlaky's algorithm is active set method, start from a primal feasible solution to construct dual feasible solution which is complimentary to the primal feasible solution. Terlaky [11] proposed an algorithm which does not require the enlargement of the basic table as Frank-Wolfe [4] method. Wolfe Philip [13] has given algorithm which based on fairly simple modification of simplex method and converges in finite number of iterations. Dantzig [3] suggestion is to choose that entering vector corresponding to which $z_{j}-c_{j}$ is most negative. Khobragade et al. [7] suggestion is to choose that entering vector corresponding to which $\frac{\left(z_{j}-c_{j}\right)}{\sum x_{i}}$ is most negative, where $\sum x_{i}$ is the sum of corresponding column to each $z_{j}-c_{j}$.

In this paper, an attempt has been made to solve quadratic programming problem (QPP) by new method which is an alternative for Wolfe's method. This method is different from Terlaky, Wolfe, Khobragade et al. method.

\section{AN ALTERNATIVE ALGORITHM FOR WOLFE'S MODIFIED SIMPLEX METHOD}

To find optimal solution of any QLPP by an alternative method for Wolfe's modified simplex method, algorithm is given as follows:

Step 1. First, convert the inequality constraints into equations by introducing slack-variables $\boldsymbol{y}_{\boldsymbol{i}}^{\boldsymbol{2}}(\boldsymbol{i}=$ $\mathbf{1}, \mathbf{2}, \ldots \boldsymbol{m}) \geq \mathbf{0}$ in the $\boldsymbol{i}^{\text {th }}$ constraints and the non-negative restrictions by introducing slack variables $\boldsymbol{s}_{\boldsymbol{j}}^{\mathbf{2}}(\boldsymbol{j}=$ $1,2, \ldots n) \geq 0$ in the $\boldsymbol{j}^{\text {th }}$ restrictions.

Step 2. Construct the Lagrangian function

$$
\begin{aligned}
L(x, y, s, \lambda, \mu)= & f(x) \\
& -\sum_{i=1}^{m} \lambda_{i}\left[\sum_{j=1}^{n} a_{i j} x_{j}-b_{i}+q_{i}^{2}\right] \\
& -\sum_{j=1}^{n} \mu_{j}\left[-x_{j}+s_{j}^{2}\right]
\end{aligned}
$$

Differentiating this Lagrangian function $\boldsymbol{L}(\boldsymbol{x}, \boldsymbol{y}, \boldsymbol{s}, \boldsymbol{\lambda}, \boldsymbol{\mu})$ with respect to the components of $\boldsymbol{x}, \boldsymbol{y}, \boldsymbol{s}, \boldsymbol{\lambda}, \boldsymbol{\mu}$ and equating the first order partial derivatives to zero, derive Kuhn-Tucker conditions from the resulting equations. 
Step 3. Introduce non-negative artificial variables $\boldsymbol{a}_{\boldsymbol{j}}, \boldsymbol{j}=$ $\mathbf{1}, \mathbf{2}, \ldots, \boldsymbol{n})$ in the Kuhn-Tucker conditions

$$
c_{j}+\sum_{k=1}^{n} c_{j k} x_{k}-\sum_{i=1}^{m} \lambda_{i} a_{i j}+\mu_{j}=0
$$

for $\boldsymbol{j}=\mathbf{1}, \mathbf{2}, \ldots, \boldsymbol{n}$ and construct an objective function

$$
Z=a_{1}+a_{2}+\cdots+a_{n}
$$

Step 4. Obtain the initial basic feasible solution to the LPP:

$$
\operatorname{Min} \boldsymbol{Z}=\boldsymbol{a}_{1}+\boldsymbol{a}_{2}+\cdots+\boldsymbol{a}_{\boldsymbol{n}}
$$

Subject to the constraints:

$$
\begin{gathered}
\sum_{k=1}^{n} c_{j k} x_{k}-\sum_{i=1}^{m} \lambda_{i} a_{i j}+\mu_{j}=-c_{j} ;(j=1,2, \ldots, n) \\
\sum_{j=1}^{n} a_{i j} x_{j}+q_{i}^{2}=b_{i} ;(i=1,2, \ldots, m)
\end{gathered}
$$

$$
\lambda_{i}, \mu_{j}, x_{j}, y_{i}, a_{j} \geq 0, \quad(i=1, \ldots, m ; j=1, \ldots, n)
$$

and satisfying the slackness condition:

$$
\lambda_{i} y_{i}=\mathbf{0} \text { and } \mu_{j} x_{j}=0
$$

Step 5. Solve this LPP by an alternative two-phase method. Choose greatest coefficient of decision variables.

(i) If greatest coefficient is unique, then variable corresponding to this column becomes incoming variable.

(ii) If greatest coefficient is not unique, then use tie breaking technique.

Step 6. Compute the ratio with $\boldsymbol{X}_{B}$. Choose minimum ratio, then variable corresponding to this row is outgoing variable. The element corresponding to incoming variable and outgoing variable becomes pivotal (leading) element.

Step 7. Use usual simplex method for this table and go to next step.

Step 8. Ignore corresponding row and column. Proceed to step 5 for remaining elements and repeat the same procedure either an optimal solution is obtain or there is an indication of an unbounded solution.
Step 9. If all rows and columns are ignored, current solution is an optimal solution. Thus optimum solution is obtained and which is optimum solution of given QPP also.

\section{SOLVED PROBLEMS}

\subsection{Problem 1:}

Solve the following quadratic programming problem:

$$
\text { Maximize } Z=4 x_{1}+2 x_{2}-x_{1}^{2}-x_{2}^{2}-5
$$$$
\text { Subject to: } x_{1}+x_{2} \leq 4
$$

$$
x_{1}, x_{2} \geq 0 \text {. }
$$

Solution: First, we convert the inequality constraint into equation by introducing slack variable $s_{1}^{2}$. Also the inequality constraints $x_{1}, x_{2} \geq 0$, we convert them into equations by introducing slack variables $s_{2}^{2}$ and $s_{3}^{2}$. So the problem becomes

$$
\begin{aligned}
& \text { Maximize } Z=4 x_{1}+2 x_{2}-x_{1}^{2}-x_{2}^{2}-5 \\
& \text { Subject to: } \begin{aligned}
x_{1} & +x_{2}+s_{1}^{2}=4 \\
& -x_{1}+s_{2}^{2}=0 \\
& -x_{2}+s_{3}^{2}=0 .
\end{aligned}
\end{aligned}
$$

Now, Construct the Lagrangian function

$$
\begin{aligned}
L\left(x_{1}, x_{2}, s_{1}, s_{2}, s_{3},\right. & \left.\lambda_{1}, \lambda_{2}, \lambda_{3}\right) \\
& =\left(4 x_{1}+2 x_{2}-x_{1}^{2}-x_{2}^{2}-5\right) \\
& -\lambda_{1}\left(x_{1}+x_{2}+s_{1}^{2}-4\right)-\lambda_{2}\left(-x_{1}+s_{2}^{2}\right) \\
& -\lambda_{3}\left(-x_{2}+s_{3}^{2}\right)
\end{aligned}
$$

By Khun-Tucker conditions, we get

$$
\begin{gathered}
2 x_{1}+\lambda_{1}-\lambda_{2}=4, \quad 2 x_{2}+\lambda_{1}-\lambda_{3}=2 \\
\lambda_{1} s_{1}=\lambda_{2} s_{2}=\lambda_{3} s_{3}=0 \\
x_{1}+x_{2}+s_{1}^{2}=4, \quad-x_{1}+s_{2}^{2}=0, \quad-x_{2}+s_{3}^{2}=0
\end{gathered}
$$

where $\quad x_{1}, x_{2}, s_{1}^{2}, \lambda_{i} \geq 0, i=1, \ldots, 3 \quad$ satisfying the complementary slackness conditions

$$
\lambda_{1} s_{1}^{2}+x_{1} \lambda_{2}+x_{2} \lambda_{3}=0
$$

Now, introducing the artificial variables $a_{1}, a_{2} \geq 0$ the given QPP is equivalent to:

$$
\text { Minimize } Z=a_{1}+a_{2}
$$

Subject to: $2 x_{1}+\lambda_{1}-\lambda_{2}+a_{1}=4$

$$
\begin{array}{r}
2 x_{2}+\lambda_{1}-\lambda_{3}+a_{2}=2 \\
x_{1}+x_{2}+s_{1}^{2}=4
\end{array}
$$

where $x_{1}, x_{2}, s_{1}^{2}, a_{1}, a_{2}, \lambda_{i} \geq 0, i=1, \ldots, 3$.

Simplex table:

\begin{tabular}{|c|c|c|c|c|c|c|c|c|c|c|c|}
\hline$C_{B}$ & BVS & $X_{B}$ & $x_{1}$ & $x_{2}$ & $\lambda_{1}$ & $\lambda_{2}$ & $\lambda_{3}$ & $a_{1}$ & $a_{2}$ & $s_{1}^{2}$ & Ratio \\
\hline 1 & $a_{1}$ & 4 & $\underline{2}$ & 0 & 1 & -1 & 0 & 1 & 0 & 0 & $2 \rightarrow$ \\
\hline 1 & $a_{2}$ & 2 & 0 & 2 & 1 & 0 & -1 & 0 & 1 & 0 & - \\
\hline 0 & $S_{1}^{2}$ & 4 & 1 & 1 & 0 & 0 & 0 & 0 & 0 & 1 & 4 \\
\hline 0 & $x_{1}$ & 2 & 1 & 0 & $1 / 2$ & $-1 / 2$ & 0 & $1 / 2$ & 0 & 0 & - \\
\hline 1 & $a_{2}$ & 2 & 0 & $\mathbf{2}$ & -1 & 0 & -1 & 0 & 1 & 0 & $1 \rightarrow$ \\
\hline 0 & $S_{1}^{2}$ & 2 & 0 & 1 & $-1 / 2$ & $1 / 2$ & 0 & $-1 / 2$ & 0 & 1 & 2 \\
\hline
\end{tabular}




\begin{tabular}{|c|c|c|c|c|c|c|c|c|c|c|c|}
\hline 0 & $x_{1}$ & 2 & 1 & 0 & $1 / 2$ & $-1 / 2$ & 0 & $1 / 2$ & 0 & 0 & \\
\hline 1 & $x_{2}$ & 1 & 0 & 1 & $1 / 2$ & 0 & $-1 / 2$ & 0 & $1 / 2$ & 0 & \\
\hline 0 & $S_{1}^{2}$ & 1 & 0 & 0 & $-3 / 2$ & $1 / 2$ & $1 / 2$ & $-1 / 2$ & $-1 / 2$ & 1 & \\
\hline
\end{tabular}

Current solution is an optimal solution. $x_{1}=2, x_{2}=1$. Max. $Z=0$.

\subsection{Problem 2:}

Use Wolfe's method to solve the following quadratic programming problem:

$$
\begin{gathered}
\text { Minimize } Z=6-6 x_{1}+2 x_{1}^{2}-2 x_{1} x_{2}+2 x_{2}^{2} \\
\text { Subject to: } x_{1}+x_{2} \leq 2 \\
x_{1}, x_{2} \geq 0 .
\end{gathered}
$$

Solution: First, we convert the inequality constraint into equation by introducing slack variable $s_{1}^{2}$. Also the inequality constraints $x_{1}, x_{2} \geq 0$, we convert them into equations by introducing slack variables $s_{2}^{2}$ and $s_{3}^{2}$. So the problem becomes

Maximize $Z=-6+6 x_{1}-2 x_{1}^{2}+2 x_{1} x_{2}-2 x_{2}^{2}$

Subject to: $x_{1}+x_{2}+s_{1}^{2}=2$

$$
\begin{aligned}
& -x_{1}+s_{2}^{2}=0 \\
& -x_{2}+s_{3}^{2}=0 .
\end{aligned}
$$

Now, Construct the Lagrangian function

$$
\begin{aligned}
L\left(x_{1}, x_{2}, s_{1}, s_{2}, s_{3},\right. & \left.\lambda_{1}, \lambda_{2}, \lambda_{3}\right) \\
& =\left(-6+6 x_{1}-2 x_{1}^{2}+2 x_{1} x_{2}-2 x_{2}^{2}\right) \\
& -\lambda_{1}\left(x_{1}+x_{2}+s_{1}^{2}-2\right)-\lambda_{2}\left(-x_{1}+s_{2}^{2}\right) \\
& -\lambda_{3}\left(-x_{2}+s_{3}^{2}\right)
\end{aligned}
$$

By Khun-Tucker conditions, we get

$$
\begin{gathered}
4 x_{1}-2 x_{2}+\lambda_{1}-\lambda_{2}=6, \quad-2 x_{1}+4 x_{2}+\lambda_{1}-\lambda_{3}=0 \\
\lambda_{1} s_{1}=\lambda_{2} s_{2}=\lambda_{3} s_{3}=0 \\
x_{1}+x_{2}+s_{1}^{2}=2, \quad-x_{1}+s_{2}^{2}=0, \quad-x_{2}+s_{3}^{2}=0
\end{gathered}
$$

where $\quad x_{1}, x_{2}, s_{1}^{2}, \lambda_{i} \geq 0, i=1, \ldots, 3 \quad$ satisfying the complementary slackness conditions

$$
\lambda_{1} s_{1}^{2}+x_{1} \lambda_{2}+x_{2} \lambda_{3}=0 \text {. }
$$

Now, introducing the artificial variables $a_{1}, a_{2} \geq 0$ the given QPP is equivalent to:

Minimize $Z=a_{1}+a_{2}$

Subject to: $4 x_{1}-2 x_{2}+\lambda_{1}-\lambda_{2}+a_{1}=6$

$$
\begin{aligned}
-2 x_{1}+4 x_{2}+\lambda_{1}-\lambda_{2}+a_{2} & =0 \\
x_{1}+x_{2}+s_{1}^{2} & =2
\end{aligned}
$$

where $x_{1}, x_{2}, s_{1}^{2}, a_{1}, a_{2}, \lambda_{i} \geq 0, i=1, \ldots, 3$.

Simplex table:

\begin{tabular}{|c|c|c|c|c|c|c|c|c|c|c|c|}
\hline$C_{B}$ & BVS & $X_{B}$ & $x_{1}$ & $x_{2}$ & $\lambda_{1}$ & $\lambda_{2}$ & $\lambda_{3}$ & $a_{1}$ & $a_{2}$ & $S_{1}^{2}$ & Ratio \\
\hline 1 & $a_{1}$ & 6 & 4 & -2 & 1 & -1 & 0 & 1 & 0 & 0 & $3 / 2 \rightarrow$ \\
\hline 1 & $a_{2}$ & 0 & -2 & 4 & 1 & 0 & -1 & 0 & 1 & 0 & - \\
\hline 0 & $S_{1}^{2}$ & 2 & 1 & 1 & 0 & 0 & 0 & 0 & 0 & 1 & 2 \\
\hline 0 & $x_{1}$ & $3 / 2$ & 1 & $-1 / 2$ & $1 / 4$ & $-1 / 4$ & 0 & $1 / 4$ & 0 & 0 & - \\
\hline 1 & $a_{2}$ & 3 & 0 & 3 & $3 / 2$ & $-1 / 2$ & -1 & $1 / 2$ & 1 & 0 & $1 \rightarrow$ \\
\hline 0 & $S_{1}^{2}$ & $1 / 2$ & 0 & $3 / 2$ & $-1 / 4$ & $1 / 4$ & 0 & $-1 / 4$ & 0 & 1 & $1 / 3$ \\
\hline 0 & $x_{1}$ & $5 / 3$ & 1 & 0 & $1 / 6$ & $-1 / 6$ & 0 & $1 / 6$ & 0 & $1 / 3$ & 10 \\
\hline 1 & $a_{2}$ & 2 & 0 & 0 & 2 & -1 & -1 & 1 & 1 & -2 & $1 \rightarrow$ \\
\hline 0 & $x_{2}$ & $1 / 3$ & 0 & 1 & $-1 / 6$ & $1 / 6$ & 0 & $-1 / 6$ & 0 & $2 / 3$ & - \\
\hline 0 & $x_{1}$ & $3 / 2$ & 1 & 0 & 0 & $-1 / 2$ & $1 / 12$ & $1 / 12$ & $1 / 12$ & $1 / 2$ & \\
\hline 0 & $\lambda_{1}$ & 1 & 0 & 0 & 1 & $-1 / 2$ & $-1 / 2$ & $1 / 2$ & $1 / 2$ & -1 & \\
\hline 0 & $x_{2}$ & $1 / 2$ & 0 & 1 & 0 & $1 / 12$ & $-1 / 12$ & $-1 / 12$ & $1 / 12$ & $1 / 2$ & \\
\hline
\end{tabular}

Current solution is an optimal solution. $x_{1}=\frac{3}{2}, x_{2}=\frac{1}{2}$. Max. $Z=\frac{1}{2}$.

\subsection{Problem 3:}

Apply Wolfe's method to solve the QPP:

Maximize $Z=2 x_{1}+3 x_{2}-2 x_{1}^{2}$

Subject to: $x_{1}+4 x_{2} \leq 4$

$$
\begin{array}{r}
x_{1}+x_{2} \leq 2 \\
x_{1}, x_{2} \geq 0 .
\end{array}
$$

Solution: First, we convert the inequality constraints into equations by introducing slack variables $s_{1}^{2}$ and $s_{2}^{2}$ respectively. Also the inequality constraints $x_{1}, x_{2} \geq 0$, we convert them into equations by introducing slack variables $s_{3}^{2}$ and $s_{4}^{2}$. So the problem becomes

$$
\begin{array}{r}
\text { Maximize } Z=2 x_{1}+3 x_{2}-2 x_{1}^{2} \\
\text { Subject to: } x_{1}+4 x_{2}+s_{1}^{2}=4 \\
x_{1}+x_{2}+s_{2}^{2}=2 \\
-x_{1}+s_{3}^{2}=0 \\
-x_{2}+s_{4}^{2}=0 .
\end{array}
$$


Now, Construct the Lagrangian function

$$
\begin{aligned}
L\left(x_{1}, x_{2}, s_{1}, s_{2}, s_{3},\right. & \left.s_{4}, \lambda_{1}, \lambda_{2}, \lambda_{3}, \lambda_{4}\right) \\
& =\left(2 x_{1}+3 x_{2}-2 x_{1}^{2}\right) \\
& -\lambda_{1}\left(x_{1}+4 x_{2}+s_{1}^{2}-4\right) \\
& -\lambda_{2}\left(x_{1}+x_{2}+s_{2}^{2}-2\right)-\lambda_{3}\left(-x_{1}+s_{3}^{2}\right) \\
& -\lambda_{4}\left(-x_{2}+s_{4}^{2}\right)
\end{aligned}
$$

By Khun-Tucker conditions, we get

$$
\begin{gathered}
4 x_{1}+\lambda_{1}+\lambda_{2}-\lambda_{3}=2, \quad 4 \lambda_{1}+\lambda_{2}-\lambda_{4}=3 \\
\lambda_{1} s_{1}=\lambda_{2} s_{2}=\lambda_{3} s_{3}=\lambda_{4} s_{4}=0 \\
x_{1}+4 x_{2}+s_{1}^{2}=4, \quad x_{1}+x_{2}+s_{2}^{2}=2,
\end{gathered}
$$

$$
-x_{1}+s_{3}^{2}=0, \quad-x_{2}+s_{4}^{2}=0
$$

where $\quad x_{1}, x_{2}, s_{1}^{2}, s_{2}^{2}, \lambda_{i} \geq 0, i=1, \ldots, 4 \quad$ satisfying the complementary slackness conditions

$$
\lambda_{1} s_{1}^{2}+\lambda_{2} s_{2}^{2}+x_{1} \lambda_{3}+x_{2} \lambda_{4}=0 .
$$

Now, introducing the artificial variables $a_{1}, a_{2} \geq 0$ the given QPP is equivalent to:

Minimize $Z=a_{1}+a_{2}$

Subject to: $4 x_{1}+\lambda_{1}+\lambda_{2}-\lambda_{3}+a_{1}=2$

$$
\begin{array}{r}
4 \lambda_{1}+\lambda_{2}-\lambda_{4}+a_{2}=3 \\
x_{1}+4 x_{2}+s_{1}^{2}=4 \\
x_{1}+x_{2}+s_{2}^{2}=2
\end{array}
$$

where $x_{1}, x_{2}, s_{1}^{2}, s_{2}^{2}, a_{1}, a_{2}, \lambda_{i} \geq 0, i=1, \ldots, 4$.

\section{Simplex table:}

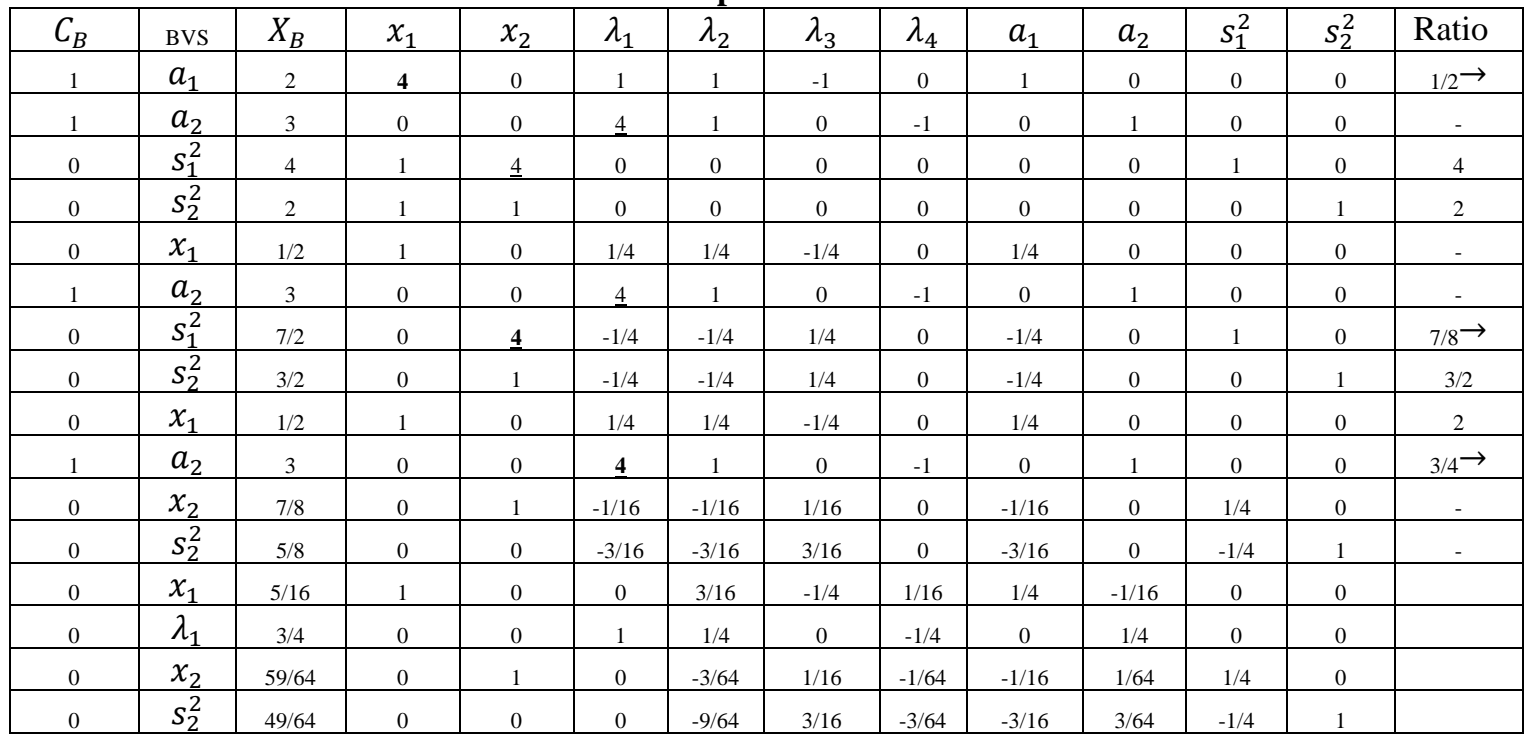

Current solution is an optimal solution. $x_{1}=\frac{5}{16}, x_{2}=\frac{59}{16}$. Max. $Z=3.19$.

\subsection{Problem 4:}

Solve by Wolfe's method:

$$
\begin{gathered}
\text { Maximize } Z=2 x_{1}+x_{2}-x_{1}^{2} \\
\text { Subject to: } 2 x_{1}+3 x_{2} \leq 6 \\
2 x_{1}+x_{2} \leq 4 \\
x_{1}, x_{2} \geq 0
\end{gathered}
$$

Solution: First, we convert the inequality constraints into equations by introducing slack variables $s_{1}^{2}$ and $s_{2}^{2}$ respectively. Also the inequality constraints $x_{1}, x_{2} \geq 0$, we convert them into equations by introducing slack variables $s_{3}^{2}$ and $s_{4}^{2}$. So the problem becomes

$$
\begin{array}{r}
\text { Maximize } Z=2 x_{1}+x_{2}-x_{1}^{2} \\
\text { Subject to: } 2 x_{1}+3 x_{2}+s_{1}^{2}=6 \\
2 x_{1}+x_{2}+s_{2}^{2}=4 \\
-x_{1}+s_{3}^{2}=0 \\
-x_{2}+s_{4}^{2}=0
\end{array}
$$

Now, Construct the Lagrangian function

$$
\begin{aligned}
L\left(x_{1}, x_{2}, s_{1}, s_{2}, s_{3},\right. & \left.s_{4}, \lambda_{1}, \lambda_{2}, \lambda_{3}, \lambda_{4}\right) \\
& =\left(2 x_{1}+x_{2}-x_{1}^{2}\right) \\
& -\lambda_{1}\left(2 x_{1}+3 x_{2}+s_{1}^{2}-6\right) \\
& -\lambda_{2}\left(2 x_{1}+x_{2}+s_{2}^{2}-4\right) \\
& -\lambda_{3}\left(-x_{1}+s_{3}^{2}\right)-\lambda_{4}\left(-x_{2}+s_{4}^{2}\right)
\end{aligned}
$$

By Khun-Tucker conditions, we get

$$
\begin{gathered}
2 x_{1}+2 \lambda_{1}+2 \lambda_{2}-\lambda_{3}=2, \quad 3 \lambda_{1}+\lambda_{2}-\lambda_{4}=1 \\
\lambda_{1} s_{1}=\lambda_{2} s_{2}=\lambda_{3} s_{3}=\lambda_{4} s_{4}=0 \\
2 x_{1}+3 x_{2}+s_{1}^{2}=6, \quad 2 x_{1}+x_{2}+s_{2}^{2}=4, \\
-x_{1}+s_{3}^{2}=0, \quad-x_{2}+s_{4}^{2}=0
\end{gathered}
$$


where $x_{1}, x_{2}, s_{1}^{2}, s_{2}^{2}, \lambda_{i} \geq 0, i=1, \ldots, 4 \quad$ satisfying the complementary slackness conditions

$$
\lambda_{1} s_{1}^{2}+\lambda_{2} s_{2}^{2}+x_{1} \lambda_{3}+x_{2} \lambda_{4}=0 .
$$

Now, introducing the artificial variables $a_{1}, a_{2} \geq 0$ the given QPP is equivalent to:

$$
\text { Minimize } Z=a_{1}+a_{2}
$$

Subject to: $2 x_{1}+2 \lambda_{1}+2 \lambda_{2}-\lambda_{3}+a_{1}=2$

$$
\begin{array}{r}
3 \lambda_{1}+\lambda_{2}-\lambda_{4}+a_{2}=1 \\
2 x_{1}+3 x_{2}+s_{1}^{2}=6 \\
2 x_{1}+x_{2}+s_{2}^{2}=4
\end{array}
$$

where $x_{1}, x_{2}, s_{1}^{2}, s_{2}^{2}, a_{1}, a_{2}, \lambda_{i} \geq 0, i=1, \ldots, 4$.

\begin{tabular}{|c|c|c|c|c|c|c|c|c|c|c|c|c|c|}
\hline \multicolumn{11}{|c|}{ Simplex table: } \\
\hline$C_{B}$ & BVS & $X_{B}$ & $x_{1}$ & $x_{2}$ & $\lambda_{1}$ & $\lambda_{2}$ & $\lambda_{3}$ & $\lambda_{4}$ & $a_{1}$ & $a_{2}$ & $s_{1}^{2}$ & $s_{2}^{2}$ & Ratio \\
\hline 1 & $a_{1}$ & 2 & 2 & 0 & 2 & 2 & -1 & 0 & 1 & 0 & 0 & 0 & 1 \\
\hline 1 & $a_{2}$ & 1 & 0 & 0 & $\mathbf{3}$ & 1 & 0 & -1 & 0 & 1 & 0 & 0 & $1 / 3 \rightarrow$ \\
\hline 0 & $S_{1}^{2}$ & 6 & 2 & $\underline{3}$ & 0 & 0 & 0 & 0 & 0 & 0 & 1 & 0 & - \\
\hline 0 & $S_{2}^{2}$ & 4 & 2 & 1 & 0 & 0 & 0 & 0 & 0 & 0 & 0 & 1 & - \\
\hline 1 & $a_{1}$ & $4 / 3$ & 2 & 0 & 0 & $4 / 3$ & -1 & $2 / 3$ & 1 & $-2 / 3$ & 0 & 0 & - \\
\hline 0 & $\lambda_{1}$ & $1 / 3$ & 0 & 0 & 1 & $1 / 3$ & 0 & $-1 / 3$ & 0 & $1 / 3$ & 0 & 0 & - \\
\hline 0 & $S_{1}^{2}$ & 6 & 2 & $\mathbf{3}$ & 0 & 0 & 0 & 0 & 0 & 0 & 1 & 0 & $2 \rightarrow$ \\
\hline 0 & $S_{2}^{2}$ & 4 & 2 & 1 & 0 & 0 & 0 & 0 & 0 & 0 & 0 & 1 & 4 \\
\hline 1 & $a_{1}$ & $4 / 3$ & $\mathbf{2}$ & 0 & 0 & $4 / 3$ & -1 & $2 / 3$ & 1 & $-2 / 3$ & 0 & 0 & $2 / 3 \rightarrow$ \\
\hline 0 & $\lambda_{1}$ & $1 / 3$ & 0 & 0 & 1 & $1 / 3$ & 0 & $-1 / 3$ & 0 & $1 / 3$ & 0 & 0 & - \\
\hline 0 & $x_{2}$ & 2 & $2 / 3$ & 1 & 0 & 0 & 0 & 0 & 0 & 0 & $1 / 3$ & 0 & 3 \\
\hline 0 & $S_{2}^{2}$ & 2 & $4 / 3$ & 0 & 0 & 0 & 0 & 0 & 0 & 0 & $-1 / 3$ & 1 & $3 / 2$ \\
\hline 0 & $x_{1}$ & $2 / 3$ & 1 & 0 & 0 & $2 / 3$ & $-1 / 2$ & $1 / 3$ & $1 / 2$ & $-1 / 3$ & 0 & 0 & \\
\hline 0 & $\lambda_{1}$ & $1 / 3$ & 0 & 0 & 1 & $1 / 3$ & 0 & $-1 / 3$ & 0 & $1 / 3$ & 0 & 0 & \\
\hline 0 & $x_{2}$ & $14 / 9$ & 0 & 1 & 0 & $-4 / 9$ & $1 / 3$ & $-2 / 9$ & $-1 / 3$ & $2 / 9$ & $1 / 3$ & 0 & \\
\hline 0 & $S_{2}^{2}$ & $10 / 9$ & 0 & 0 & 0 & $-8 / 9$ & $2 / 3$ & $-4 / 9$ & $-2 / 3$ & $4 / 9$ & $-1 / 3$ & 1 & \\
\hline
\end{tabular}

Current solution is an optimal solution. $x_{1}=\frac{2}{3}, x_{2}=\frac{14}{9}$. Max. $Z=\frac{22}{9}$.

\subsection{Problem 5:}

Solve the following quadratic programming problem:

$$
\begin{gathered}
\text { Minimize } Z=-4 x_{1}+x_{1}^{2}-2 x_{1} x_{2}+2 x_{2}^{2} \\
\text { Subject to: } 2 x_{1}+x_{2} \leq 6 \\
x_{1}-4 x_{2} \leq 0 \\
x_{1}, x_{2} \geq 0
\end{gathered}
$$

Solution: First, we convert the inequality constraints into equations by introducing slack variables $s_{1}^{2}$ and $s_{2}^{2}$ respectively. Also the inequality constraints $x_{1}, x_{2} \geq 0$, we convert them into equations by introducing slack variables $s_{3}^{2}$ and $s_{4}^{2}$. So the problem becomes

$$
\begin{gathered}
\text { Maximize } Z=4 x_{1}-x_{1}^{2}+2 x_{1} x_{2}-2 x_{2}^{2} \\
\text { Subject to: } 2 x_{1}+x_{2}+s_{1}^{2}=6 \\
\qquad \begin{aligned}
x_{1}-4 x_{2}+s_{2}^{2} & =0 \\
-x_{1}+s_{3}^{2} & =0 \\
-x_{2}+s_{4}^{2} & =0 .
\end{aligned}
\end{gathered}
$$

Now, Construct the Lagrangian function

$$
\begin{aligned}
L\left(x_{1}, x_{2}, s_{1}, s_{2}, s_{3},\right. & \left.s_{4}, \lambda_{1}, \lambda_{2}, \lambda_{3}, \lambda_{4}\right) \\
& =\left(4 x_{1}-x_{1}^{2}+2 x_{1} x_{2}-2 x_{2}^{2}\right) \\
& -\lambda_{1}\left(2 x_{1}+x_{2}+s_{1}^{2}-6\right) \\
& -\lambda_{2}\left(x_{1}-4 x_{2}+s_{2}^{2}\right)-\lambda_{3}\left(-x_{1}+s_{3}^{2}\right) \\
& -\lambda_{4}\left(-x_{2}+s_{4}^{2}\right)
\end{aligned}
$$

By Khun-Tucker conditions, we get

$$
\begin{gathered}
2 x_{1}-2 x_{2}+2 \lambda_{1}+\lambda_{2}-\lambda_{3}=4, \\
-2 x_{1}+4 x_{2}+\lambda_{1}-4 \lambda_{2}-\lambda_{4}=0 \\
\lambda_{1} s_{1}=\lambda_{2} s_{2}=\lambda_{3} s_{3}=\lambda_{4} s_{4}=0 \\
2 x_{1}+x_{2}+s_{1}^{2}=6, \quad x_{1}-4 x_{2}+s_{2}^{2}=0, \\
-x_{1}+s_{3}^{2}=0, \quad-x_{2}+s_{4}^{2}=0
\end{gathered}
$$

where $x_{1}, x_{2}, s_{1}^{2}, s_{2}^{2}, \lambda_{i} \geq 0, i=1, \ldots, 4$ satisfying the complementary slackness conditions

$$
\lambda_{1} s_{1}^{2}+\lambda_{2} s_{2}^{2}+x_{1} \lambda_{3}+x_{2} \lambda_{4}=0 .
$$

Now, introducing the artificial variables $a_{1}, a_{2} \geq 0$ the given QPP is equivalent to: Subject to:

$$
\text { Minimize } Z=a_{1}+a_{2}
$$

$$
\begin{aligned}
2 x_{1}-2 x_{2}+2 \lambda_{1}+\lambda_{2}-\lambda_{3}+a_{1} & =4 \\
-2 x_{1}+4 x_{2}+\lambda_{1}-4 \lambda_{2}-\lambda_{4}+a_{2} & =0 \\
2 x_{1}+x_{2}+s_{1}^{2} & =6 \\
x_{1}-4 x_{2}+s_{2}^{2} & =0
\end{aligned}
$$

where $x_{1}, x_{2}, s_{1}^{2}, s_{2}^{2}, a_{1}, a_{2}, \lambda_{i} \geq 0, i=1, \ldots, 4$. 
Simplex table:

\begin{tabular}{|c|c|c|c|c|c|c|c|c|c|c|c|c|}
\hline$C_{B}$ & $\mathrm{BVS}$ & $X_{B}$ & $x_{1}$ & $x_{2}$ & $\lambda_{1}$ & $\lambda_{2}$ & $\lambda_{3}$ & $\lambda_{4}$ & $a_{1}$ & $a_{2}$ & $S_{1}^{2}$ & $S_{2}^{2}$ \\
\hline 1 & $a_{1}$ & 4 & 2 & -2 & 2 & 1 & -1 & 0 & 1 & 0 & 0 & 0 \\
\hline 1 & $a_{2}$ & 0 & -2 & 4 & 1 & -4 & 0 & -1 & 0 & 1 & 0 & 0 \\
\hline 0 & $S_{1}^{2}$ & 6 & 2 & 1 & 0 & 0 & 0 & 0 & 0 & 0 & 1 & 0 \\
\hline 0 & $S_{2}^{2}$ & 0 & 1 & -4 & 0 & 0 & 0 & 0 & 0 & 0 & 0 & 1 \\
\hline 1 & $a_{1}$ & 4 & 1 & 0 & $5 / 4$ & -1 & -1 & $-1 / 2$ & 1 & $1 / 2$ & 0 & 0 \\
\hline 0 & $x_{2}$ & 0 & $-1 / 2$ & 1 & $1 / 4$ & -1 & 0 & $-1 / 4$ & 0 & $1 / 4$ & 0 & 0 \\
\hline 0 & $S_{1}^{2}$ & 6 & $\mathbf{5} / \mathbf{2}$ & 0 & $-1 / 4$ & 1 & 0 & $1 / 4$ & 0 & $-1 / 4$ & 1 & 0 \\
\hline 0 & $S_{2}^{2}$ & 0 & -1 & 0 & 1 & -4 & 0 & -1 & 0 & 1 & 0 & 1 \\
\hline 1 & $a_{1}$ & $8 / 5$ & 0 & 0 & $\mathbf{1 3} / 5$ & $-7 / 5$ & -1 & $-3 / 5$ & 1 & $3 / 5$ & $-2 / 5$ & 0 \\
\hline 0 & $x_{2}$ & $6 / 5$ & 0 & 1 & $1 / 5$ & $-4 / 5$ & 0 & $-1 / 5$ & 0 & $1 / 5$ & $1 / 5$ & 0 \\
\hline 0 & $x_{1}$ & $12 / 5$ & 1 & 0 & $-1 / 10$ & $2 / 5$ & 0 & $1 / 10$ & 0 & $-1 / 10$ & $2 / 5$ & 0 \\
\hline 0 & $S_{2}^{2}$ & $12 / 5$ & 0 & 0 & $9 / 10$ & $-18 / 5$ & 0 & $-9 / 10$ & 0 & $9 / 10$ & $2 / 5$ & 1 \\
\hline 0 & $\lambda_{1}$ & $8 / 13$ & 0 & 0 & 1 & $-7 / 13$ & $-5 / 13$ & $-3 / 13$ & $5 / 13$ & $1 / 26$ & 0 & 0 \\
\hline 0 & $x_{2}$ & $14 / 13$ & 0 & 1 & 0 & $-9 / 13$ & $1 / 13$ & $-2 / 13$ & $-1 / 13$ & $5 / 26$ & 0 & 0 \\
\hline 0 & $x_{1}$ & $32 / 13$ & 1 & 0 & 0 & $9 / 26$ & $-1 / 26$ & $1 / 13$ & $1 / 26$ & $-5 / 52$ & 1 & 0 \\
\hline 0 & $S_{2}^{2}$ & $24 / 13$ & 0 & 0 & 0 & $-81 / 26$ & $9 / 26$ & $-9 / 13$ & $-9 / 26$ & $45 / 52$ & 0 & 1 \\
\hline
\end{tabular}

Current solution is an optimal solution. $x_{1}=\frac{32}{13}, x_{2}=\frac{14}{13}$. Min. $Z=-\frac{88}{13}$.

\section{CONCLUSION}

An alternative method for Wolfe's method to obtain the solution of quadratic programming problems has been derived. An algorithm that performs well on one type of the problem may perform poorly on problem with a different structure. A number of algorithms have been developed, each applicable to specific type of NPPP only. The numbers of application of non-linear programming are very large and it is not possible to give a comprehensive survey of all of them. However, an efficient method for the solution of general NLPP is still. This technique is useful to apply on numerical problems, reduces the labour work and save valuable time.

\section{REFERENCES}

[1]. Beale E.M.L., On quadratic programming, Naval Research Logistics Quarterly 6, 1969, pp. 227-244.

[2]. Boot J.C.G., Quadratic Programming, North-Holland, Amsterdam, 1964.

[3]. Dantzig G. B., Linear Programming and Extensions, Princeton University Press, Princeton, 1963.

[4]. Frank M. and Wolfe P.: An Algorithm for Quadratic Programming, Naval Research Logistics Quarterly 3, March-June 1956, pp. 95-110.

[5]. Gupta P. K., Man Mohan.: Problems in Operation Research methods and solutions, Sultan Chand and Sons, Educational Publications, New Delhi.

[6]. Hildreth C.: A Quadratic Programming Procedure, Naval Research Logistics Quarterly 4, March 1957,79-85.

[7]. Khobragade N. W., Lamba N. K. and Khot P. G.: Alternative Approach to Wolfe's Modified Simplex Method for Quadratic Programming Problems, Int. J. Latest Trend Math, Vol-2, No.1, March-2012.

[8]. Kuhn H. W. and Tucker A. W.: Nonlinear Programming, Proceedings of the Second Berkerley
Symposium on Mathematical Statistics and Probability, 1951, 481-492.

[9]. Ritter K.: A dual quadratic programming algorithm, University of Wisconsin-Madison, Mathematics Research Center, Technical Summary Report No. 2733, August 1984. [10]. Sharma S. D.: Operation Research, Kedar Nath Ram Nath, 132, R. G. Road, Meerut-250001 (U.P.), India.

[11]. Terlaky T: A New Algorithm for Quadratic Programming, European Journal of Operation Research, Vol.32,1987, pp. 294-301, North-Holland.

[12]. Van de Panne, C., Methods for Linear and Quadratic Programming, North-Holland, Amsterdam, 1975.

[13]. Wolfe Philip: The Simplex Method for Quadratic Programming, The Econometric Society, Econometrica, Vol. 27, No. 3, Jul.1959, pp. 382-398.

\section{BIOGRAPHIES}

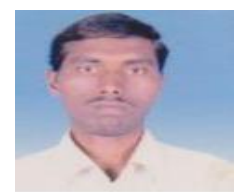

Mr. Tanaji S. Pawar, Research student, Department of mathematics, Dr. Babasaheb Ambedkar Marathwada University, Aurangabad.

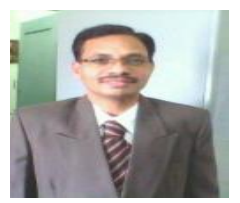

Dr. K. P. Ghadle for being M.Sc in Maths, he attained Ph.D. He has been teaching since 1994. At present he is working as Associate Professor. Achieved excellent experiences in Research for 15 years in the area of Boundary value problems and its application. Published more than 50 research papers in reputed journals. Four students awarded Ph.D. Degree, three students awarded M. Phil and four students working for award of Ph.D. Degree under their guidance. 